\title{
Baixa temperatura noturna e deficiência hídrica na fotossíntese de cana-de-açúcar
}

\author{
Daniela Favero São Pedro Machado( ${ }^{(1)}$, Ana Maria Magalhães Andrade Lagôa(1), Rafael Vasconcelos Ribeiro(2), \\ Paulo Eduardo Ribeiro Marchiori(1), Ricardo Silvério Machado(1) e Eduardo Caruso Machado ${ }^{(1)}$
}

(1)Instituto Agronômico, Caixa Postal 28, CEP 13012-970 Campinas, SP. E-mail: danifavero@yahoo.com.br, alagoa@iac.sp.gov.br,
marchiori.paulo@gmail.com, machado_rs@yahoo.com.br, caruso@iac.sp.gov.br (2) Universidade Estadual de Campinas, Instituto de Biologia,
Departamento de Biologia Vegetal, Rua Monteiro Lobato, no-255, CEP 13083-862 Campinas, SP. E-mail: rvr@unicamp.br

Resumo - O objetivo deste trabalho foi avaliar as respostas fotossintéticas da cana-de-açúcar aos efeitos simultâneos e isolados de baixa temperatura noturna (TN) e deficiência hídrica (DH). Após 128 dias do plantio, as plantas da cultivar IACSP94-2094 foram submetidas aos tratamentos: controle, sem DH e com TN de $20^{\circ} \mathrm{C}$ (TN20); com DH e TN de $20^{\circ} \mathrm{C}$ (DH/TN20); sem DH e com TN de $12^{\circ} \mathrm{C}(\mathrm{TN} 12)$; e com DH e TN de $12^{\circ} \mathrm{C}$ (DH/TN12) por cinco dias. Após o período de tratamento, as plantas foram irrigadas e submetidas à $\mathrm{TN}$ de $20^{\circ} \mathrm{C}$ por mais quatro dias, para recuperação. Houve decréscimos na assimilação de $\mathrm{CO}_{2}$ em todos os tratamentos. A recuperação total da assimilação de $\mathrm{CO}_{2}$ foi observada apenas nas plantas do tratamento TN12. A ocorrência simultânea da baixa temperatura noturna e da deficiência hídrica causou dano acentuado e persistente na condutância estomática, na capacidade máxima da ribulose-1,5-bisfosfato carboxilase, no transporte aparente de elétrons, no fator de eficiência e na eficiência operacional do fotossistema II, o que resultou em limitações difusivas, bioquímicas e fotoquímicas da fotossíntese das plantas de cana-de-açúcar.

Termos para indexação: Saccharum, assimilação de $\mathrm{CO}_{2}$, PEPcase, resfriamento, Rubisco.

\section{Low night temperature and water deficit on photosynthesis of sugarcane}

\begin{abstract}
The objective of this work was to evaluate the photosynthetic responses of sugarcane to the simultaneous and isolated effects of low night temperature (TN) and water deficit (DH). After 128 days of planting, plants of the cultivar IACSP94-2094 were subjected to the following treatments: control, without DH and with TN of $20^{\circ} \mathrm{C}(\mathrm{TN} 20)$; with DH and TN of $20^{\circ} \mathrm{C}(\mathrm{DH} / \mathrm{TN} 20)$; without $\mathrm{DH}$ and with TN of $12^{\circ} \mathrm{C}(\mathrm{TN} 12)$; and with $\mathrm{DH}$ and $\mathrm{TN}$ of $12^{\circ} \mathrm{C}$ (DH/TN12). After the period of treatment, plants were irrigated and subjected to $\mathrm{TN}$ of $20^{\circ} \mathrm{C}$ for four more days, for recovery. There were decreases in $\mathrm{CO}_{2}$ assimilation in all treatments. Total recovery of $\mathrm{CO}_{2}$ assimilation was observed only in plants from the treatment TN12. The simultaneous occurrence of low night temperature and water deficit caused a accentuated and persistent effect on stomatal conductance, on the maximum capacity of ribulose-1,5-bisphosphate carboxylase, on the electron transport rate, on the efficiency factor, and on the operational efficiency of photosystem II, which resulted in diffusive, biochemical, and photochemical limitations of photosynthesis of sugarcane plants.
\end{abstract}

Index terms: Saccharum, $\mathrm{CO}_{2}$ assimilation, PEPcase, cooling, Rubisco.

\section{Introdução}

A cultura de cana-de-açúcar (Saccharum spp.) tem importância mundial como fonte de alimento e de energia. No Brasil, o cultivo dessa espécie tem aumentado em áreas marginais, onde há variações anuais acentuadas de temperatura e da disponibilidade hídrica (Rolim et al., 2007). Episódios de baixa temperatura noturna ocorrem com certa frequência e causam danos que podem afetar a produtividade das plantas (Soares-Cordeiro et al., 2010; Sales et al., 2012). Nas regiões subtropicais, a ocorrência de baixas temperaturas noturnas é comum no final do inverno, quando há deficiência hídrica no solo e dias com temperaturas relativamente altas.

Plantas têm tolerância variável à baixa temperatura, e, em espécies tropicais sensíveis ao resfriamento, podem ocorrer danos mesmo quando estas são expostas a temperaturas superiores à de congelamento do tecido (Sage \& Kubien, 2007; Kakani et al., 2008). Folhas de plantas expostas ao resfriamento mostram inibição da fotossíntese, translocação mais lenta de carboidratos, menor respiração, inibição do metabolismo de proteínas, geração de espécies reativas de oxigênio, crescimento lento e murcha foliar. Estas modificações 
são causadas por disfunções em processos metabólicos promovidos por alterações das propriedades da membrana, mudanças na estrutura de proteínas e nas interações entre macromoléculas, e pela inibição de reações enzimáticas (Carmo-Silva et al., 2008; Kakani et al., 2008; Soares-Cordeiro et al., 2010; Liu et al., 2012). Em condições de baixa temperatura noturna, observou-se decréscimo na fotossíntese em espécies $\mathrm{C}_{4}$ (milho e gramíneas), o que pode ser atribuído tanto a fatores difusivos quanto metabólicos (Kakani et al., 2008; Soares-Cordeiro et al., 2010).

Além das condições térmicas, a deficiência hídrica do solo constitui um dos estresses mais limitantes da produtividade vegetal. A deficiência hídrica tem impacto variável na produtividade agrícola dependendo da fase fenológica em que ocorre (Inman-Bamber \& Smith, 2005; Machado et al., 2009), e há genótipos de cana-de-açúcar que mostram diferentes capacidades de recuperação no crescimento após eventos de seca (Landell et al., 2004; Machado et al., 2009). Sob deficiência hídrica, as plantas apresentam alterações morfofisiológicas, tais como: enrolamento e alteração do ângulo da folha; redução da área foliar, da transpiração, da condutância estomática, da fotossíntese e da condutividade hidráulica das raízes; modificação da atividade de enzimas do metabolismo de nitrogênio e de carbono; e mudanças nos teores de antioxidantes (Ghannoum, 2009; Machado et al., 2009; Lopes et al., 2011; Ribeiro et al., 2013). A fotossíntese decresce inicialmente em razão do fechamento dos estômatos e, com o agravamento da deficiência hídrica, também se evidenciam limitações metabólicas (Ghannoum et al., 2003; Carmo-Silva et al., 2008; Ghannoum, 2009; Lopes et al., 2011).

Estudos sobre os danos causados pela baixa temperatura noturna e pela deficiência hídrica em espécies $\mathrm{C}_{3}$ são frequentes (Machado et al., 2010); porém, em espécies $\mathrm{C}_{4}$, como a cana-de-açúcar, são mais escassos (Sales et al., 2012).

O objetivo deste trabalho foi avaliar as respostas fotossintéticas da cana-de-açúcar aos efeitos simultâneos e isolados de baixa temperatura noturna e deficiência hídrica.

\section{Material e Métodos}

Foram plantados mini toletes de cana-de-açúcar (Saccharum spp.), cultivar IACSP94-2094, em bandejas contendo substrato comercial composto de turfa de esfagno, vermiculita expandida, calcário dolomítico, gesso agrícola e fertilizante NPK (traços). Aos 35 dias após o plantio (DAP), as mudas foram transplantadas para vasos de plástico contendo 8,5 L de uma mistura na proporção 1:1:1 de terra, areia e substrato. As plantas foram mantidas em casa de vegetação, e receberam irrigação diária e aplicações de nutrientes. Estas aplicações foram parceladas em três vezes, o que totalizou, por vaso: $18,21 \mathrm{~g}$ de $\mathrm{Ca}\left(\mathrm{NO}_{3}\right)_{2}$; 5,31 g de MAP; 7,29 $\mathrm{g}$ de $\mathrm{KCl} ; 2,12 \mathrm{~g}$ de $\mathrm{MgSO}_{4}$; $0,17 \mathrm{~g}$ de $\mathrm{H}_{3} \mathrm{BO}_{3} ; 0,20 \mathrm{~g}$ de $\mathrm{MnSO}_{4} ; 0,02 \mathrm{~g}$ de $\mathrm{CuSO}_{4}$; $0,02 \mathrm{~g}$ de $\left(\mathrm{NH}_{4}\right)_{6} \mathrm{Mo}_{7} \mathrm{O}_{24} \times 4\left(\mathrm{H}_{2} \mathrm{O}\right) ; 0,09 \mathrm{~g}$ de $\mathrm{ZnSO}_{4}$; e 42,5 mL de solução de Fe-EDTA. A radiação solar variou entre 400 e $1.014 \mathrm{~W} \mathrm{~m}^{-2}$ e a temperatura do ar entre 16 e $28^{\circ} \mathrm{C}$ durante o experimento.

Aos 128 DAP, as plantas foram submetidas aos seguintes tratamentos: testemunha, com temperatura noturna (TN) de $20^{\circ} \mathrm{C}$ e irrigação (TN20); TN de $20^{\circ} \mathrm{C}$ com deficiência hídrica (DH/TN20); TN de $12^{\circ} \mathrm{C}$ com irrigação (TN12); e TN de $12^{\circ} \mathrm{C}$ com deficiência hídrica (DH/TN12). Para imposição da baixa temperatura noturna, as plantas foram transferidas para câmara de crescimento modelo PGR14 (Conviron, Winnipeg, Manitoba, Canadá) e permaneceram nessa condição durante 12 horas (das $19 \mathrm{~h}$ às $7 \mathrm{~h}$ ), sendo transferidas para a casa de vegetação no dia seguinte. As plantas foram irrigadas diariamente, tendo-se feito a reposição da água evapotranspirada, a qual foi medida por pesagens diárias dos vasos. A deficiência hídrica foi imposta pela suspensão da rega, e o substrato foi mantido com $50 \%$ da capacidade máxima de retenção de água, medida pela pesagem dos vasos (Sales et al., 2012). Os tratamentos foram aplicados pelo período de cinco dias. Na sequência, as plantas foram submetidas a um período de quatro dias de recuperação, quando a TN retornou a $20^{\circ} \mathrm{C}$ nos tratamentos TN12 e DH/ TN12, e as plantas dos tratamentos DH/TN20 e DH/ TN12 foram reidratadas. A reidratação do substrato foi realizada ao entardecer do quinto dia de tratamento, até $90 \%$ de capacidade máxima de retenção de água.

O potencial da água $\left(\psi_{\mathrm{W}}\right)$ na folha foi medido em câmara psicrométrica C-52 (Wescor, Inc., Logan, UT, EUA) e em microvoltímetro HR-33T (Wescor, Inc., Logan, UT, EUA), no modo higrométrico, às $14 \mathrm{~h}$ (Boyer, 1995).

$\mathrm{Na}$ primeira folha totalmente expandida e com a lígula aparente (folha +1 ), foram realizadas, 
simultaneamente, as medidas de trocas gasosas e fluorescência da clorofila $a$, por meio de analisador de gases por infravermelho integrado com câmara de fluorescência, modelo Li 6400-40F (Licor, Inc., Lincoln, NE, EUA). Na folha +1 de cada planta, foram realizadas curvas de resposta da assimilação de $\mathrm{CO}_{2}$ (A) e da fluorescência da clorofila $a$ pela variação da concentração intercelular de $\mathrm{CO}_{2}\left(\mathrm{C}_{\mathrm{i}}\right)$, nas condições de densidade de fluxo de fótons fotossintéticos (DFFF) de $2.000 \mu \mathrm{mol} \mathrm{m} \mathrm{m}^{-2} \mathrm{~s}^{-1}$, de temperatura foliar de $30^{\circ} \mathrm{C} \mathrm{e}$ de deficit de pressão de vapor entre a folha e o ar de $1,5 \mathrm{kPa}$. A variação de $\mathrm{C}_{\mathrm{i}}$ foi obtida pela variação da concentração de $\mathrm{CO}_{2}$ do ar $\left(\mathrm{C}_{\mathrm{ar}}\right)$ de entrada da câmara de medida, de acordo com Long \& Bernacchi (2003).

As variáveis analisadas em relação à fluorescência da clorofila $a$ foram: fluorescência mínima $\left(\mathrm{F}_{\mathrm{o}}\right)$ e máxima $\left(\mathrm{F}_{\mathrm{m}}\right)$, medidas após adaptação ao escuro por $30 \mathrm{~min}$; fluorescência no estado de equilíbrio dinâmico $\left(F^{\prime}\right)$ e máxima $\left(\mathrm{F}_{\mathrm{m}}{ }^{\prime}\right)$, medidas após adaptação à luz (Maxwell \& Johnson, 2000). A fluorescência variável máxima no escuro e à luz foram calculadas, respectivamente, por $F_{v}=F_{m}-F_{o}$ e $F_{v}{ }^{\prime}=F_{m}{ }^{\prime}-F_{o}{ }^{\prime}$, em que $F_{o}{ }^{\prime}$ representa a fluorescência mínima em folhas adaptadas à luz. O termo $F_{q}^{\prime}$ foi calculado por $F_{q}{ }^{\prime}=F_{m}{ }^{\prime}-F^{\prime}$, que representou a extinção fotoquímica da fluorescência causada pelos centros oxidados do fotossistema II (FSII) segundo Baker (2008). Dessa forma, calcularamse a eficiência quântica máxima $\left(\mathrm{F}_{\mathrm{v}} / \mathrm{F}_{\mathrm{m}}\right)$ e a eficiência operacional do FSII $\left(\mathrm{F}_{\mathrm{q}}{ }^{\prime} / \mathrm{F}_{\mathrm{m}}{ }^{\prime}\right)$, o transporte aparente de elétrons $\left(\mathrm{ETR}=\mathrm{F}_{\mathrm{q}}{ }^{\prime} / \mathrm{F}_{\mathrm{m}}{ }^{\prime} \times \mathrm{DFFF} \times 0,5 \times 0,84\right)$ e o fator de eficiência do FSII $\left(\mathrm{F}_{\mathrm{q}}{ }^{1} / \mathrm{F}_{\mathrm{v}}{ }^{\prime}\right)$ de acordo com Maxwell \& Johnson (2000) e Baker (2008), respectivamente. Para o cálculo de ETR, considerou-se uma distribuição de elétrons entre o FSI e o FSII de 0,5 e absorção de luz de 0,85 . Calculou-se, também, a eficiência quântica da fotossíntese $\left(\Phi \mathrm{CO}_{2}\right)$ pela relação $\mathrm{A} / \mathrm{DFFF}$, para cada valor de $\mathrm{C}_{\mathrm{i}}$.

A partir da curva A vs. $C_{i}$, foram calculadas: a eficiênciaaparente de carboxilação da fosfoenolpiruvato carboxilase (PEPcase, $\mathrm{V}_{\text {pmáx }}$ ), pela inclinação inicial da curva $\left(\mathrm{dA} / \mathrm{dC}_{\mathrm{i}}\right)$, em valores abaixo de $100 \mu \mathrm{mol}$ $\mathrm{mol}^{-1}$ (Caemmerer, 2000); a capacidade máxima da ribulose-1,5-bisfosfato carboxilase/oxigenasse (Rubisco, $\mathrm{V}_{\text {máx }}$ ), de acordo com Collatz et al. (1992); a assimilação de $\mathrm{CO}_{2}$ a $400 \mu \mathrm{mol} \mathrm{mol}{ }^{-1}$ no ar; e a assimilação máxima de $\mathrm{CO}_{2}$ em concentração saturante de $\mathrm{CO}_{2}\left(\mathrm{~A}_{\text {pot }}\right)$. Estimou-se a limitação metabólica $\left(\mathrm{L}_{\mathrm{M}}\right)$ pela equação $L_{M}=\left[\left(A-A^{\prime}\right) / A\right] 100$, conforme Lawlor
(2002), em que A é a assimilação de $\mathrm{CO}_{2}$ das plantas controle, e TN20 e A' são a assimilação das plantas sob estresse, medida em $\mathrm{C}_{\mathrm{i}}$ de $400 \mu \mathrm{mol} \mathrm{mol}{ }^{-1}$. As curvas A vs. $\mathrm{C}_{\mathrm{i}}$ foram realizadas no primeiro, no segundo e no quarto dias após o início dos tratamentos e no primeiro e no quarto dias após a reidratação do solo.

A variação diurna da assimilação de $\mathrm{CO}_{2}$ foi avaliada no terceiro e no quinto dias após o início dos tratamentos, tendo-se considerado a variação natural das condições atmosféricas. Os valores de DFFF foram medidos no início de cada horário de avaliação, fixados e utilizados para as demais réplicas. Calculou-se a assimilação diurna de $\mathrm{CO}_{2}\left(\mathrm{~A}_{\mathrm{i}}\right)$ a partir da integração da assimilação instantânea de $\mathrm{CO}_{2}$ ao longo do dia.

Utilizou-se o delineamento experimental inteiramente ao acaso com parcelas subdivididas no tempo, e as causas de variação foram os quatro tratamentos (TN20, DH/TN20, TN12 e DH/TN12) em cada período de avaliação. Os resultados foram submetidos à análise de variância, e as médias, obtidas de três repetições. Quando encontradas diferenças significativas, as médias foram comparadas pelo teste de Tukey, a 5\% de probabilidade.

\section{Resultados e Discussão}

A imposição do frio e do deficit hídrico causou redução significativa na assimilação diurna de $\mathrm{CO}_{2}\left(\mathrm{~A}_{\mathrm{i}}\right)$ a partir do terceiro dia de tratamento, com reduções de 24,31 e $42 \%$ nos tratamentos DH/TN20, TN12 e $\mathrm{DH} / \mathrm{TN} 12$, respectivamente, em relação à testemunha TN20 (Figura $1 \mathrm{~A}$ ). No quinto dia de tratamento, $\mathrm{A}_{\mathrm{i}}$ apresentou decréscimo mais acentuado com 71, 50 e $61 \%$ nos tratamentos DH/TN20, TN12 e DH/TN12, respectivamente, o que mostra efeito cumulativo conforme o tempo de estresse. Os menores valores observados em $\mathrm{A}_{\mathrm{i}}$, no quinto dia, decorreram da menor incidência de radiação solar no período.

Tanto A quanto $\mathrm{g}_{\mathrm{s}}$ foram menores nos tratamentos, em comparação à testemunha, no segundo dia de baixa temperatura noturna e restrição hídrica (Figura $1 \mathrm{~B}$ e C). Até o segundo dia, o efeito isolado do frio noturno (TN12) e o simultâneo do frio noturno mais deficit hídrico (DH/TN12) sobre a fotossíntese (A) foram mais acentuados que o da deficiência hídrica (DH/TN20) (Figura $1 \mathrm{~B}$ ). Após o segundo dia, a fotossíntese, nas plantas submetidas à deficiência hídrica (DH/TN20) e ao frio noturno e à deficiência hídrica (DH/TN12), 

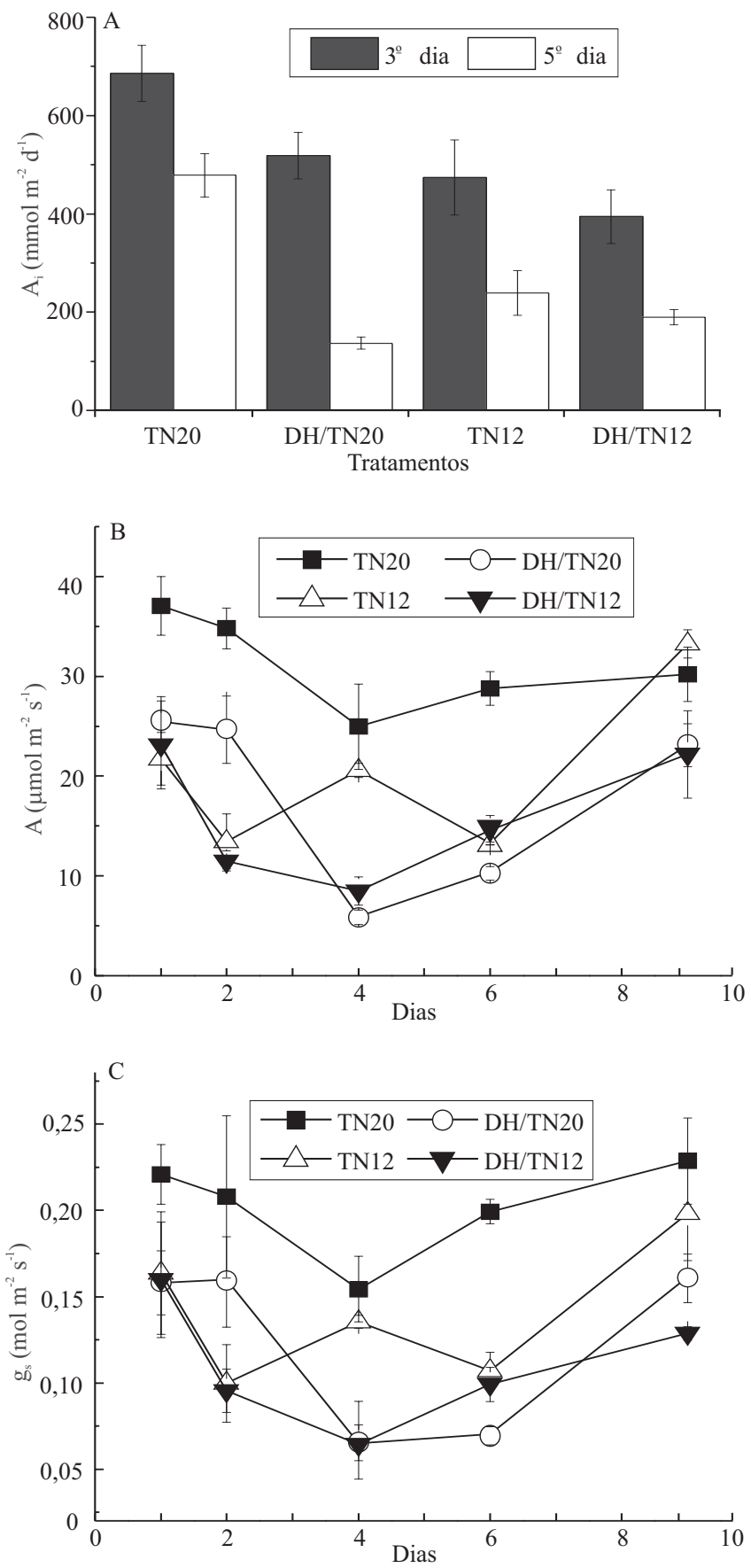

Figura 1. A, assimilação diurna de $\mathrm{CO}_{2}\left(\mathrm{~A}_{\mathrm{i}}\right)$ de cana-de-açúcar. As colunas indicam média de três repetições ( \pm erro padrão). $\mathrm{B}$, assimilação de $\mathrm{CO}_{2}(\mathrm{~A})$. C , condutância estomática $\left(\mathrm{g}_{\mathrm{s}}\right)$ em cana-de-açúcar. Tratamentos: TN20, temperatura noturna de $20^{\circ} \mathrm{C}$ com irrigação; DH/TN20, temperatura noturna de $20^{\circ} \mathrm{C}$ sem irrigação; TN12, temperatura noturna de $12^{\circ} \mathrm{C}$ com irrigação; e DH/TN12, temperatura noturna de $12^{\circ} \mathrm{C}$ sem irrigação. Dias 1 a 5, período de aplicação dos tratamentos; dias 5 a 9, período de recuperação com retorno da irrigação das plantas e temperatura noturna de $20^{\circ} \mathrm{C}$. continuou menor em comparação a TN20, tendo atingido valores menores que as plantas submetidas ao tratamento TN12. Após quatro dias da reidratação do substrato e do retorno a TN20, apenas as plantas TN12 apresentaram A semelhante à da testemunha, tendo persistido o efeito do frio associado ao deficit hídrico sobre A nas plantas DH/TN12 e DH/TN20.

O decréscimo da fotossíntese observado em plantas tanto em baixa temperatura noturna quanto sob deficiência hídrica, ou sob a combinação de ambos os estresses, pode ser atribuído a fatores difusivos, metabólicos ou fotoquímicos (Ghannoum et al., 2003; Ghannoum, 2009; Machado et al., 2009, 2010). No segundo dia de tratamento em plantas sob TN12, DH/TN12 e DH/TN20, a g foi menor (Figura $1 \mathrm{C}$ ) e houve alta correlação entre $\mathrm{A}$ e $\mathrm{g}_{\mathrm{s}}$ nas plantas estudadas $\left(A=-0,57+174,22 g_{s}, R^{2}=0,91, p<0,01\right)$, o que indica que o fechamento parcial dos estômatos causou limitações à fotossíntese. A queda em $\mathrm{g}_{\mathrm{s}}$ em plantas submetidas à baixa temperatura noturna (TN12 e DH/ TN12) ocorreu possivelmente em razão da redução da condutância hidráulica ocasionada pelo decréscimo da permeabilidade do protoplasma (Machado et al., 2010), uma vez que não houve variação de $\psi_{\mathrm{w} 14}$ nestes tratamentos. $\mathrm{O}$ fechamento de estômatos ocasionado pela redução da umidade do substrato está relacionado com a diminuição do $\psi_{\mathrm{W} 14}$ em DH/TN20 (Figura 2 A), com a condutividade hidráulica e com o transporte de sinais bioquímicos, como o transporte e a redistribuição do ácido abscísico resultante da alcalinização da seiva do xilema, desde as raízes até as folhas (Ghannoum et al., 2009; Machado et al., 2009; 2010).

Assim como observado para $\mathrm{g}_{\mathrm{s}}$, $\mathrm{E}$ foi menor nos tratamentos TN12, DH/TN20 e DH/TN12, em comparação ao controle, após quatro dias dos tratamentos (Figura 2 B). Somente as plantas do tratamento $\mathrm{DH} / \mathrm{TN} 20$ tiveram menor $\psi_{\mathrm{w} 14}$ que a testemunha (Figura $2 \mathrm{~A}$ ). Esse resultado é intrigante, pois, em relação às plantas $\mathrm{DH} / \mathrm{TN} 12$, também sob estresse hídrico, $\mathrm{g}_{\mathrm{s}}$ e E foram semelhantes (Figura $1 \mathrm{~B}$; Figura 2 B). No quarto dia de recuperação, nas plantas DH/TN20 e DH/TN12, a reidratação proporcionou recuperação parcial de $g_{s}$ e E (Figura $1 \mathrm{C}$; Figura $2 \mathrm{~B}$ ). Somente TN12 recuperou totalmente $g_{s}$ e E. Em todos os casos, o $\psi_{\mathrm{w}_{14}}$ recuperou-se totalmente no quarto dia após a irrigação, na temperatura noturna de $20^{\circ} \mathrm{C}$.

As $\mathrm{A}_{\text {pot }}$ dos tratamentos TN12, DH/TN20 e DH/ TN12 foram menores que a da testemunha a partir do 
primeiro dia. No segundo dia, as plantas que foram submetidas a TN12 e a DH/TN12 apresentaram $\mathrm{A}_{\text {pot }}$ menor que as submetidas a DH/TN20, o que sugere efeito mais intenso da baixa $\mathrm{TN}$ do que da $\mathrm{DH}$ no início do estresse. No quinto dia, as $\mathrm{A}_{\text {pot }}$ de DH/TN20 e DH/TN12 foram as menores, enquanto as plantas TN12 apresentaram aclimatação. A $\mathrm{A}_{\text {pot }}$ é medida em

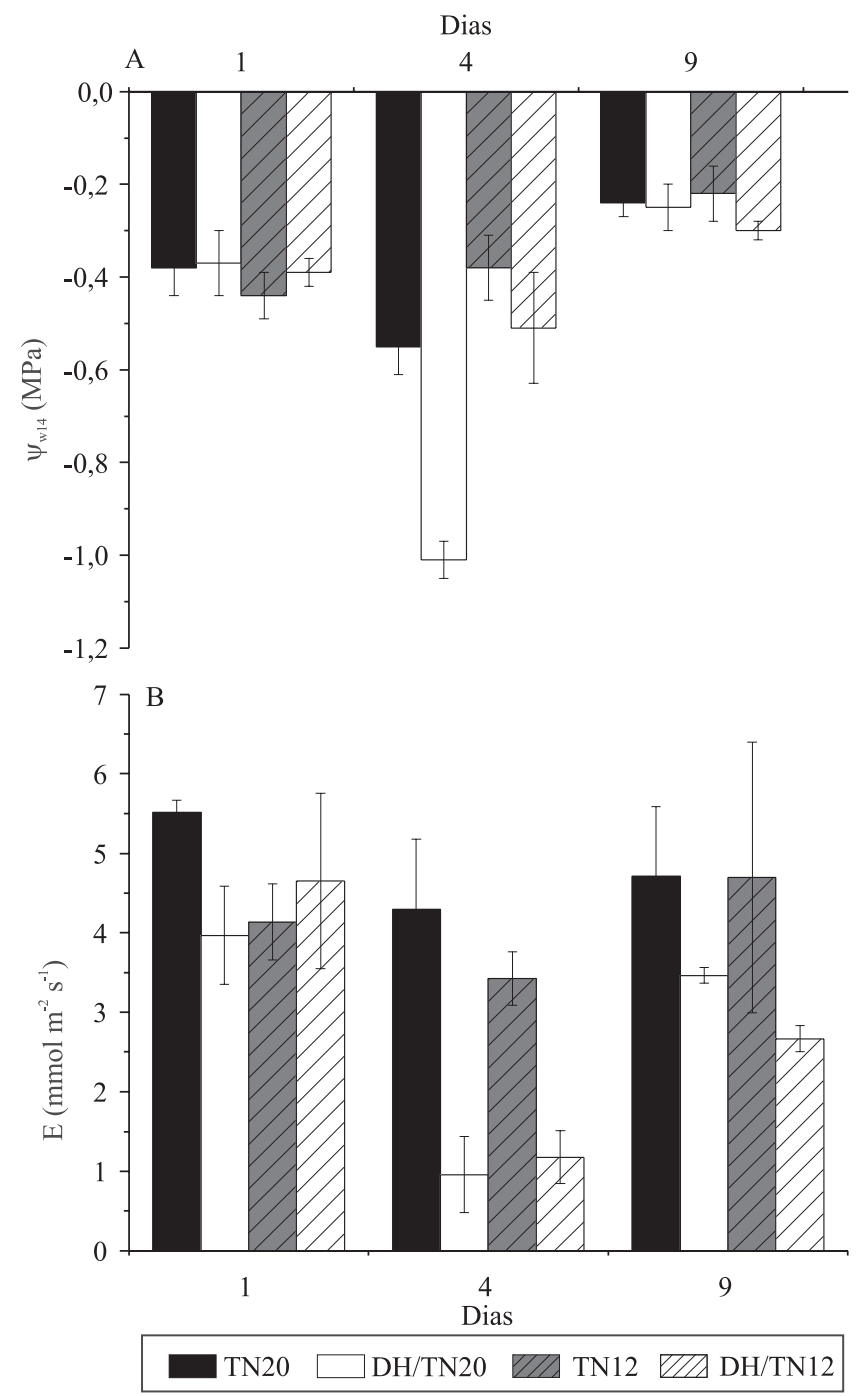

Figura 2. A, potencial da água na folha $\left(\Psi_{\mathrm{W}_{14}}\right)$; B, transpiração (E) em cana-de-açúcar, cultivar IACSP94-2094, submetida aos tratamentos: TN20, temperatura noturna de $20^{\circ} \mathrm{C}$ com irrigação; DH/TN20, temperatura noturna de $20^{\circ} \mathrm{C}$ sem irrigação; TN12, temperatura noturna de $12^{\circ} \mathrm{C}$ com irrigação; e DH/TN12, temperatura noturna de $12^{\circ} \mathrm{C}$ sem irrigação. As colunas indicam média de três repetições ( \pm erro padrão). Dias 1 a 5, período de aplicação dos tratamentos; dias 5 a 9, período de recuperação com retorno da irrigação das plantas e temperatura noturna de $20^{\circ} \mathrm{C}$. condições de saturação de $\mathrm{CO}_{2} \mathrm{e}$, portanto, desconsidera limitações difusivas. Assim, os valores inferiores em relação às plantas TN20 indicam limitações metabólicas (Figura 3 A). Em DH/TN20 e DH/TN12, os valores de $A_{\text {pot }}$ foram menores que os dos outros tratamentos no final do período de recuperação, o que sugere que as limitações metabólicas ainda persistiram nestes dois tratamentos, com destaque para DN/TN12 que foi menor que DH/TN20. De fato, o decréscimo da fotossíntese foi associado ao aumento significativo da limitação metabólica $\left(\mathrm{L}_{\mathrm{M}}\right)$ nas plantas que sofreram restrição hídrica (DH/TN20 e DH/TN12) e que não apresentaram redução de $\mathrm{L}_{\mathrm{M}}$ após a reidratação do solo (Figura 3 B). Também, neste caso, a $\mathrm{L}_{\mathrm{M}}$ de DH/TN12 ficou maior que a de $\mathrm{DH} / \mathrm{TN} 20$ na recuperação.

$\mathrm{O}$ ciclo fotossintético $\mathrm{C}_{4}$ da cana-de-açúcar caracteriza-se por adaptações celulares, bioquímicas e fisiológicas. $\mathrm{O} \quad \mathrm{CO}_{2}$ atmosférico é inicialmente fixado por fosfoenolpiruvato-carboxilase (PEPcase), em ácido $\mathrm{C}_{4}$, nas células do mesofilo. Os ácidos $\mathrm{C}_{4}$ são transportados para as células da bainha do feixe vascular (BFV), onde são descarboxilados, e o $\mathrm{CO}_{2}$ liberado entra no metabolismo $\mathrm{C}_{3}$ via ribulose-1,5-bifosfato (RuBP) carboxilase/oxigenase (Rubisco) confinada nas células da BFV. Este mecanismo concentra $\mathrm{CO}_{2}$ próximo à enzima Rubisco, o que permite sua maior atividade como carboxilase e resulta em maior eficiência do uso de água e de nitrogênio e na ausência de fotorrespiração (Sage \& Kubien, 2007). Dessa forma, a $\mathrm{L}_{\mathrm{M}}$ da fotossíntese pode estar relacionada a alterações na atividade enzimática da PEPcase e da Rubisco, com regeneração dos substratos das enzimas e transporte de elétrons (Ripley et al., 2007; Carmo-Silva et al., 2008; Ghannoum, 2009; Soares-Cordeiro et al., 2010). A eficiência aparente de carboxilação da PEPcase $\left(\mathrm{V}_{\text {pmáx }}\right)$ foi menor do que a da testemunha, no primeiro dia, em todos os tratamentos. No entanto, houve aclimatação das plantas no início do período de recuperação, ou seja, os valores de $\mathrm{V}_{\text {pmáx }}$ se igualaram aos verificados em TN20 (Figura 3 C). Alguns estudos também mostraram a redução na atividade da PEPcase com a diminuição do conteúdo de água no solo (Du et al., 1996; Markelz et al., 2011) e com a baixa temperatura noturna (Kakani et al., 2008; Soares-Cordeiro et al., 2010).

$\mathrm{V}_{\text {máx }}$ foi menor que a testemunha em todos os tratamentos no primeiro dia, sendo mais acentuada em TN12 e DH/TN12, no segundo dia, e em DH/TN20 e 
DH/TN12 no quarto dia de tratamento. Nas plantas do tratamento TN12, foi observada a aclimatação de $\mathrm{V}_{\text {máx }}$. Os valores de $\mathrm{V}_{\text {máx }}$ foram maiores que os dos outros tratamentos e próximos ao da testemunha no quarto dia de tratamento, e semelhantes ao da testemunha no final do período de recuperação (Figura $3 \mathrm{D}$ ). As plantas DH/TN12 não apresentaram recuperação de $\mathrm{V}_{\text {máx }}$ ao término do período de recuperação (Figura 3
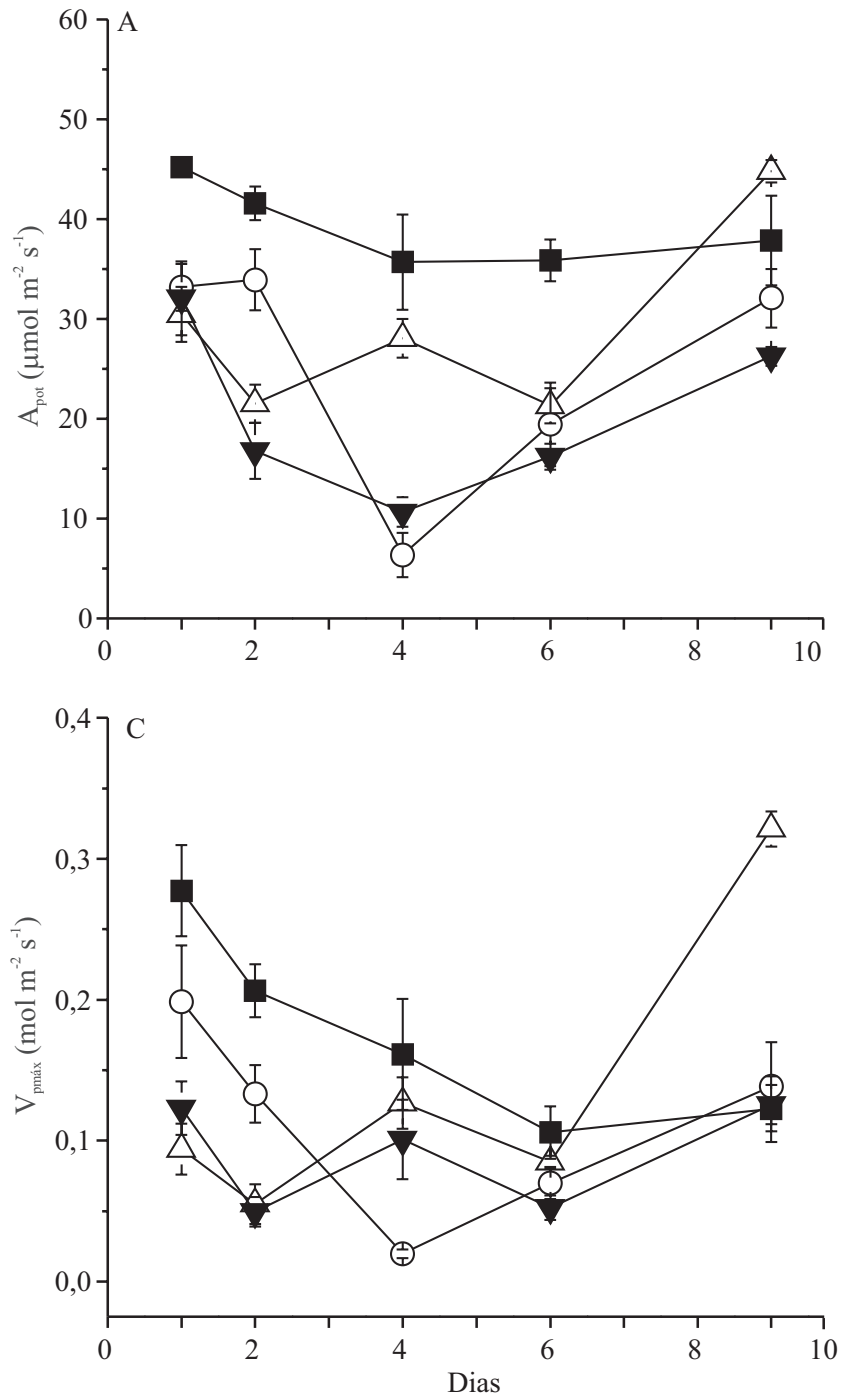

D). As reduções de $V_{\text {pmáx }}$ e $V_{\text {máx }}$ indicam limitações bioquímicas do metabolismo fotossintético $\mathrm{C}_{4}$ da cana-de-açúcar em condições de baixa temperatura noturna e deficit hídrico. A atividade da Rubisco foi mais sensível aos tratamentos, em especial nas plantas submetidas ao deficit hídrico (DH/TN20 e DH/TN12), mesmo após a reidratação, quando apresentavam $\mathrm{L}_{\mathrm{M}}$ de aproximadamente $25 \%$, em razão dos baixos valores
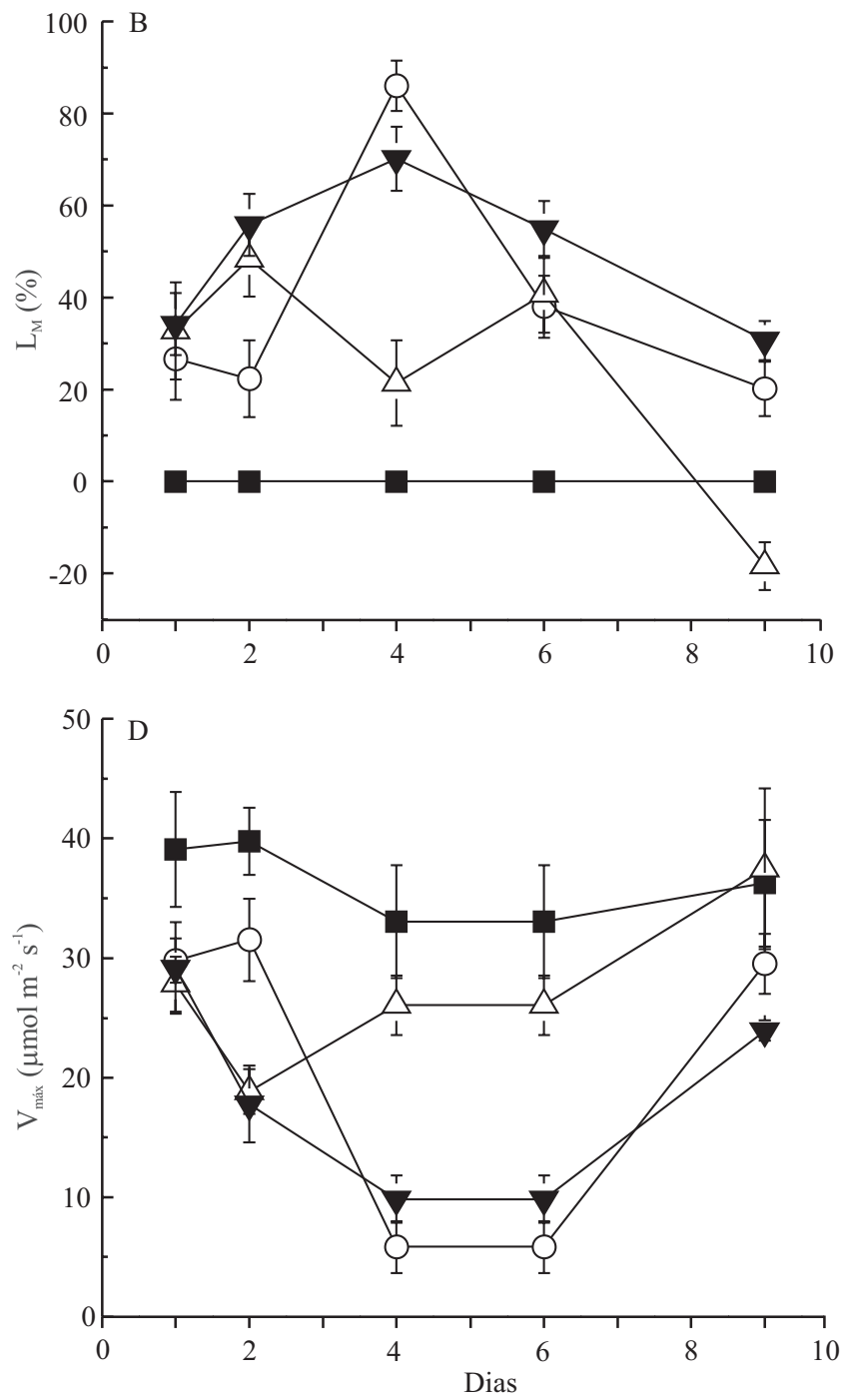

$$
\text { TN20 } \multimap-\text { DH/TN20 } \longrightarrow \text { TN12 } \longrightarrow \text { DH/TN12 }
$$

Figura 3. $A$, assimilação máxima em $\mathrm{CO}_{2}$ saturante $\left(\mathrm{A}_{\mathrm{pot}}\right)$; $\mathrm{B}$, limitação metabólica $\left(\mathrm{L}_{\mathrm{M}}\right)$; $\mathrm{C}$, eficiência de carboxilação da PEPcase $\left(\mathrm{V}_{\text {pmáx }}\right)$; e $\mathrm{D}$, eficiência máxima da Rubisco $\left(\mathrm{V}_{\text {máx }}\right)$ em cana-de-açúcar, cultivar IACSP94-2094, submetida aos tratamentos: TN20, temperatura noturna de $20^{\circ} \mathrm{C}$ com irrigação; DH/TN20, temperatura noturna de $20^{\circ} \mathrm{C}$ sem irrigação; TN12, temperatura noturna de $12^{\circ} \mathrm{C}$ com irrigação; e DH/TN12, temperatura noturna de $12^{\circ} \mathrm{C}$ sem irrigação. Os símbolos indicam média de três repetições ( \pm erro padrão). Dias 1 a 5, período de aplicação dos tratamentos; dias 5 a 9, período de recuperação com retorno da irrigação das plantas e temperatura noturna de $20^{\circ} \mathrm{C}$. 
de $V_{\text {máx }}$ (Figura 3 A e D). No quarto dia de recuperação, a $\mathrm{V}_{\text {máx }}$ de DH/TN12 foi menor que a de DH/TN20 (Figura $3 \mathrm{D}$ ).

Os tratamentos não causaram variações significativas em $F_{v} / F_{m}$, o que sugere que não houve fotoinibição da fotossíntese (Figura $4 \mathrm{~A}$ ). No entanto, ETR e $\mathrm{F}_{\mathrm{q}}{ }^{\prime} / \mathrm{F}_{\mathrm{m}}$ 'foram menores no primeiro dia dos tratamentos do que no controle (Figura $4 \mathrm{~B} \mathrm{e} \mathrm{C}$ ). Apenas as plantas do tratamento DH/TN12 não recuperaram ETR e
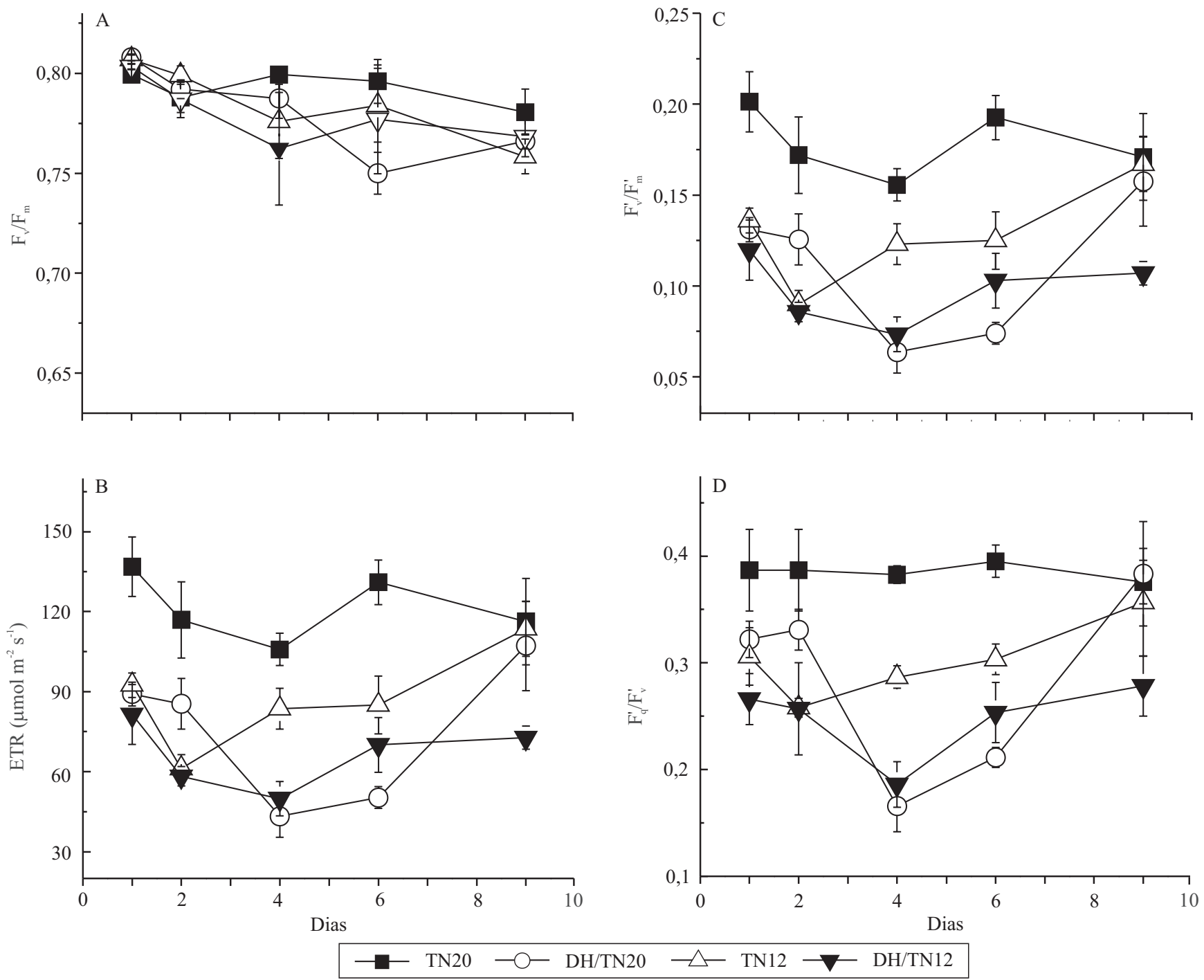

Figura 4. A, eficiência quântica máxima do fotossistema II $\left(\mathrm{F}_{\mathrm{v}} / \mathrm{F}_{\mathrm{m}}\right)$; B, eficiência operacional do FSII (Fq'/Fm'); C, transporte aparente de elétrons (ETR); e D, fator de eficiência do FSII (Fq'/Fv') em cana-de-açúcar, cultivar IACSP94-2094, submetida aos tratamentos: TN20, temperatura noturna de $20^{\circ} \mathrm{C}$ com irrigação; DH/TN20, temperatura noturna de $20^{\circ} \mathrm{C}$ sem irrigação; TN12, temperatura noturna de $12^{\circ} \mathrm{C}$ com irrigação; e DH/TN12, temperatura noturna de $12^{\circ} \mathrm{C}$ sem irrigação. Os símbolos indicam média de três repetições ( \pm erro-padrão). Dias 1 a 5 , período de aplicação dos tratamentos; dias 5 a 9 , período de recuperação com retorno da irrigação das plantas e temperatura noturna de $20^{\circ} \mathrm{C}$. 
temperatura e deficit hídrico (Baker, 2008). Isso reflete em acúmulo de $\mathrm{Q}_{\mathrm{A}}$ em estado reduzido, indicado pela diminuição de $\mathrm{F}_{\mathrm{q}}{ }^{\prime} / \mathrm{F}_{\mathrm{v}}{ }^{\prime}$, resultante da significativa redução da assimilação de $\mathrm{CO}_{2}$ em DH/TN20, TN12 e DH/TN12.

A diminuição de $\mathrm{g}_{\mathrm{s}}$ causa menor fluxo de $\mathrm{CO}_{2}$ para o mesofilo foliar e pode causar redução na concentração de $\mathrm{CO}_{2}$ junto à Rubisco e estimular a atividade da oxigenase, ou seja, a fotorrespiração (Carmo-Silva et al., 2008). De fato, no presente trabalho, houve queda de $\mathrm{C}_{\mathrm{i}}$ somente em DH/TN20 e no quarto dia de estresse $\left(\mathrm{C}_{\mathrm{i}}=115 \pm 25 \mu \mathrm{mol} \mathrm{mol}^{-1} \mathrm{em} \mathrm{DH} \mathrm{e} \mathrm{C}_{\mathrm{i}}=176 \pm 19 \mu \mathrm{mol} \mathrm{mol}^{-1}\right.$ nos demais tratamentos). A relação entre $\Phi \mathrm{CO}_{2} \mathrm{e} \mathrm{F}_{\mathrm{q}}{ }^{\prime} / \mathrm{F}_{\mathrm{m}}{ }^{\prime}$ não foi modificada pelos tratamentos (Figura 5), o que sugere que não houve aumento na atividade de drenos alternativos de elétrons, como fotorrespiração e reação de Mehler (Ghannoum, 2009), e sim decréscimo no uso da energia em reações fotoquímicas da fotossíntese (Figura $4 \mathrm{C}$ e D; Figura 5). Este resultado é indicativo de que, apesar de ter ocorrido menor fluxo de $\mathrm{CO}_{2}$ em decorrência da diminuição de $\mathrm{g}_{\mathrm{s}}$, o mecanismo de

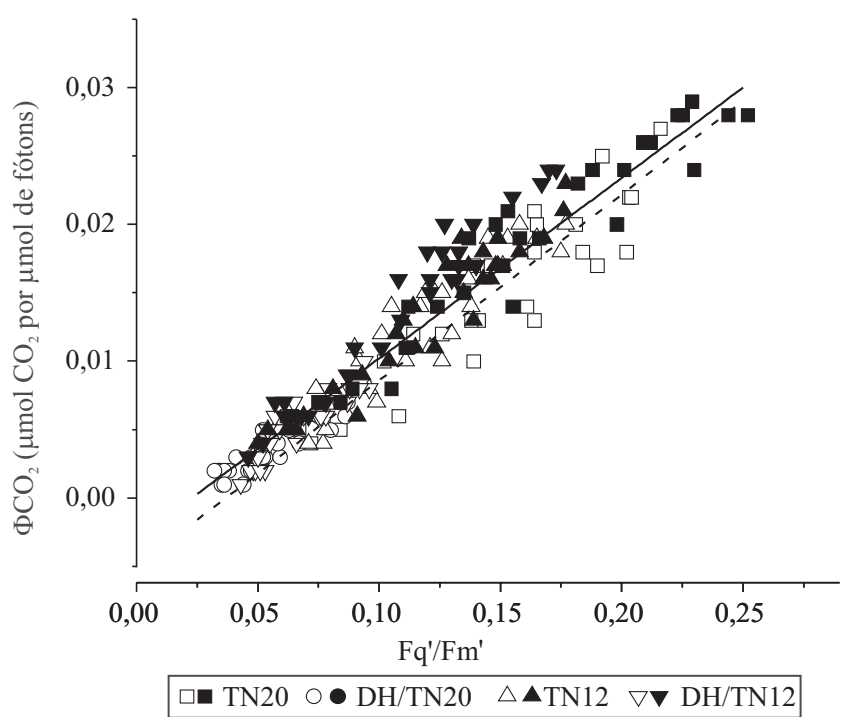

Figura 5. Relação entre eficiência quântica de $\mathrm{CO}_{2}$ $\left(\Phi \mathrm{CO}_{2}\right)$ e eficiência operacional do fotossistema II (Fq'/Fm') em cana-de-açúcar, cultivar IACSP94-2094, submetida aos tratamentos: TN20, temperatura noturna de $20^{\circ} \mathrm{C}$ com irrigação; DH/TN20, temperatura noturna de $20^{\circ} \mathrm{C}$ sem irrigação; TN12, temperatura noturna de $12^{\circ} \mathrm{C}$ com irrigação; e DH/TN12, temperatura noturna de $12^{\circ} \mathrm{C}$ sem irrigação, um dia (símbolos cheios e linha, $\left.\Phi \mathrm{CO}_{2}=-0,003+0,132 \times \mathrm{Fq}^{\prime} / \mathrm{Fm}^{\prime}, \mathrm{R}^{2}=0,93, \mathrm{p}<0,01\right)$ e quatro dias após os tratamentos (símbolos vazios e linha tracejada, $\left.\Phi \mathrm{CO}_{2}=-0,005+0,136 \times \mathrm{Fq}^{\prime} / \mathrm{Fm}^{\prime}, \mathrm{R}^{2}=0,87, \mathrm{p}<0,01\right)$. concentração de $\mathrm{CO}_{2}$ da cana-de-açúcar nos tratamentos avaliados é efetivo em limitar a fotorrespiração (Carmo-Silva et al., 2008).

\section{Conclusões}

1. A assimilação diurna de $\mathrm{CO}_{2}$ em plantas de cana-de-açúcar decresce em função da baixa temperatura noturna e da deficiência hídrica, e esta associada à diminuição da condutância estomática (fator difusivo), à redução na atividade da PEPcase e da Rubisco (fatores bioquímicos), e à menor atividade fotoquímica.

2. A ocorrência simultânea de baixa temperatura noturna e deficiência hídrica em cana-de-açúcar interfere na recuperação fotossintética das plantas.

\section{Agradecimentos}

Ao Conselho Nacional de Desenvolvimento Científico e Tecnológico (CNPq), pela concessão de bolsas; à Coordenação de Aperfeiçoamento de Pessoal de Nível Superior (Capes), pela concessão de bolsa; e à Fundação de Amparo à Pesquisa do Estado de São Paulo (Fapesp), pelo financiamento da pesquisa (Processo 2008/57495-3).

\section{Referências}

BAKER, N.R. Chlorophyll fluorescence: a probe of photosynthesis in vivo. Annual Review of Plant Biology, v.59, p.89-113, 2008. DOI: 10.1146/annurev.arplant.59.032607.092759.

BOYER, J.S. Measuring the water status of plants and soils. San Diego: Academic Press, 1995. 178p.

CAEMMERER, S. von. Biochemical models of leaf photosynthesis. Melbourne: CSIRO, 2000. 165p.

CARMO-SILVA, A.E.; POWERS, S.J.; KEYS, A.J.; ARRABAÇA, M.C.; PARRY, M.A. Photorespiration in $\mathrm{C} 4$ grasses remains slow under drought conditions. Plant, Cell and Environment, v.31, p.925-940, 2008. DOI: 10.1111/j.1365-3040.2008.01805.x.

COLlATZ, G.J.; RIBAS-CARBO, M.; BERRY, J.A. Coupled photosynthesis-stomatal conductance model for leaves of $\mathrm{C} 4$ plants. Australian Journal of Plant Physiology, v.19, p.519-538, 1992. DOI: 10.1071/PP9920519.

DU, Y.C.; KAWAMITSU, Y.; NOSE, A.; HIYANE, S.; MURAYAMA, S.; WASANO, K.; UCHIDA, Y. Effects of water stress on carbon exchange rate and activities of photosynthetic enzymes in leaves of sugarcane (Saccharum sp.). Australian Journal of Plant Physiology, v.23, p.719-726, 1996. DOI: 10.1071/PP9960719. 
GHANNOUM, O. C4 photosynthesis and water stress. Annals of Botany, v.103, p.635-644, 2009. DOI: 10.1093/aob/mcn093.

GHANNOUM, O.; CONROY, J.P.; DRISCOLL, S.P.; PAUL, M.J.; FOYER, C.H.; LAWLOR, D.W. Nonstomatal limitations are responsible for drought-induced photosynthetic inhibition in four $\mathrm{C}_{4}$ grasses. New Phytologist, v.159, p.599-608, 2003. DOI: 10.1046/j.1469-8137.2003.00835.x.

INMAN-BAMBER, N.G.; SMITH, D.M. Water relations in sugarcane and response to water deficits. Field Crops Research, v.92, p.185-202, 2005. DOI: 10.1016/j.fcr.2005.01.023.

KAKANI, V.G.; SURABHI, G.K.; REDDY, K.R. Photosynthesis and fluorescence responses of $\mathrm{C} 4$ plant Andropogon gerardii acclimated to temperature and carbon dioxide. Photosynthetica, v.46, p.420-430, 2008. DOI: 10.1007/s11099-008-0074-0.

LANDELL, M.G. de A.; CAMPANA, M.P.; FIGUEIREDO, P.; SILVA, M. de A.; VASCONCELOS, A.C.M. de: BIDOIA, M.A.P.; XAVIER, M.A.; DINARDO-MIRANDA, L.L.; PRADO, H. do; ROSSETTO, R.; SANOS, A. da S. dos; CAVICHIOLI, J.C.; MARTINS, A.L.M.; KANTHACK, R.A.D.; VEIGA FILHO, A. de A.; SILVA, D.N. da. Variedades de cana-de-açúcar para o Centro-Sul do Brasil. Campinas: IAC, 2004. 32p. (IAC. Boletim técnico, 195).

LAWLOR, D.W. Limitation to photosynthesis in water-stressed leaves: stomata vs. metabolism and the role of ATP. Annals of Botany, v.89, p.871-885, 2002. DOI: 10.1093/aob/mcf110.

LIU, Y.F.; QI, M.F.; LI, T.L. Photosynthesis, photoinhibition, and antioxidant system in tomato leaves stressed by low night temperature and their subsequent recovery. Plant Science, v.196, p.8-17, 2012. DOI: 10.1016/j.plantsci.2012.07.005.

LONG, S.P.; BERNACCHI, C.J. Gas exchange measurements, what can they tell us about the underlying limitations to photosynthesis? Procedures and sources of error. Journal of Experimental Botany, v.54, p.2393-2401, 2003. DOI: 10.1093/jxb/erg262.

LOPES, M.S.; ARAUS, J.L.; VAN HEERDEN, P.D.R.; FOYER, C.H. Enhancing drought tolerance in C4 crops. Journal of Experimental Botany, v.62, p.3135-3153, 2011. DOI: 10.1093/ jxb/err105.

MACHADO, D.F.S.P.; MACHADO, E.C.; MACHADO, R.S.; RIBEIRO, R.V. Efeito da baixa temperatura noturna e do porta-enxerto na variação diurna das trocas gasosas e na atividade fotoquímica de laranjeira 'Valência'. Revista Brasileira de Fruticultura, v.32, p.351-359, 2010. DOI: 10.1590/ S0100-29452010005000064.
MACHADO, R.S.; RIBEIRO, R.V.; MARCHIORI, P.E.R.; MACHADO, D.F.S.P.; MACHADO, E.C.; LANDELL, M.G. de A. Respostas biométricas e fisiológicas ao déficit hídrico em cana-de-açúcar em diferentes fases fenológicas. Pesquisa Agropecuária Brasileira, v.44, p.1575-1582, 2009. DOI: 10.1590/ S0100-204X2009001200003.

MARKELZ, R.J.C.; STRELLNER, R.S.; LEAKEY, A.D.B. Impairment of $\mathrm{C} 4$ photosynthesis by drought is exacerbated by limiting nitrogen and ameliorated by elevated $\left[\mathrm{CO}_{2}\right]$ in maize. Journal of Experimental Botany, v.62, p.3235-3246, 2011. DOI: 10.1093/jxb/err056.

MAXWELL, K.; JOHNSON, G.N. Chlorophyll fluorescence - a practical guide. Journal of Experimental Botany, v.51, p.659-668, 2000. DOI: 10.1093/jexbot/51.345.659.

RIBEIRO, R.V.; MACHADO, R.S.; MACHADO, E.C.; MACHADO, D.F.S.P.; MAGALHÃES FILHO, J.R.; LANDELL, M.G.A. Revealing drought-resistance and productive patterns in sugarcane genotypes by evaluating both physiological responses and stalk yield. Experimental Agriculture, v.49, p.212-224, 2013. DOI: $10.1017 /$ S0014479712001263.

RIPLEY, B.S.; GILBERT, M.E.; IBRAHIM, D.G.; OSBORNE, C.P. Drought constraints on $\mathrm{C} 4$ photosynthesis: stomatal and metabolic limitations in $\mathrm{C} 3$ and $\mathrm{C} 4$ subspecies of Alloteropsis semialata. Journal of Experimental Botany, v.58, p.1351-1363, 2007. DOI: $10.1093 / \mathrm{jxb} / \mathrm{erl302}$.

ROLIM, G. de S.; CAMARGO, M.B.P. de; LANIA, D.G.; MORAES, J.F.L. de. Classificação climática de Köppen e de Thornthwaite e sua aplicabilidade na determinação de zonas agroclimáticas para o Estado de São Paulo. Bragantia, v.66, p.711-720, 2007. DOI: 10.1590/S0006-87052007000400022.

SAGE, R.F.; KUBIEN, D.S. The temperature response of $\mathrm{C}_{3}$ and $\mathrm{C}_{4}$ photosynthesis. Plant, Cell and Environment, v.30, p.1086-1106, 2007. DOI: 10.1111/j.1365-3040.2007.01682.x.

SALES, C.R.G.; RIBEIRO, R.V.; MACHADO, D.F.S.P.; MACHADO, R.S.; DOVIS, V.L.; LAGÔA, A.M.M.A. Trocas gasosas e balanço de carboidratos em plantas de cana-de-açúcar sob condições de estresses radiculares. Bragantia, v.71, p.319-327, 2012. DOI: 10.1590/S0006-87052012000300001.

SOARES-CORDEIRO, A.S.; DRISCOLL, S.P.; ARRABAÇA, M.C.; FOYER, C.H. Dorsoventral variations in dark chilling effects on photosynthesis and stomatal function in Paspalum dilatatum leaves. Journal of Experimental Botany, v.62, p.687-699, 2010. DOI: $10.1093 /$ jxb/erq302.

Recebido em 28 de dezembro de 2012 e aprovado em 3 de maio de 2013 